\title{
Towards in-fiber silicon photonics
}

\author{
N. Healy ${ }^{1, *}$, P.J.A. Sazio ${ }^{1}$, P. Mehta ${ }^{1}$, L. Shen ${ }^{1}$, T.D. Day ${ }^{2}$, J.V. Badding ${ }^{2}$, A.C. Peacock ${ }^{1}$ \\ 1. Optoelectronics Research Centre, University of Southampton, Southampton SO17 1BJ, UK \\ 2. Department of Chemistry and Materials Research Institute, Pennsylvania State University 16802 PA, USA \\ *nvh@orc.soton.ac.uk
}

\begin{abstract}
The state of the art of silicon optical fibers fabricated via the high pressure chemical deposition technique will be reviewed. The optical transmission properties of step index silicon optical fibers will be presented, including investigations of the nonlinearities that can be used for all-optical signal processing. In addition, alternative complex fiber geometries that permit sophisticated control of the propagating light will be introduced.
\end{abstract} (C)2012 Optical Society of America

OCIS codes: $060.2270,060.2290,060.4370$

\section{Introduction}

In the last decade, silicon waveguides have proven an excellent platform for nonlinear optical signal processing and frequency generation. Silicon's large Kerr nonlinearity and high refractive index allow for tight mode confinement and high energy densities that have enabled the realisation of a number of all-optical devices, such as optical regenerators, modulators and switches. More recently, the silicon optical fiber has emerged as a complementary platform that can make use of the functionality of the material in a geometry that is compatible with conventional fiber infrastructures [1]. In this paper we review our progress in characterizing the nonlinear transmission properties of hydrogenated amorphous silicon (a-Si:H) core fibers fabricated using a high pressure chemical deposition (HPCVD) technique [2]. This material is growing in importance for nonlinear photonics as its Kerr nonlinearity is around twice that of crystalline silicon and its fabrication costs are significantly lower. The higher nonlinear refractive index has enabled us to observe substantial spectral broadening despite our fibers having relatively large cores (a few micron), and we have recently extended our characterizations from telecoms wavelengths, across the two-photon absorption (TPA) edge, and towards the mid-infrared regime where the nonlinear absorption becomes negligible.

Furthermore, an advantage of our deposition technique is that we can fabricate optical fibers that have a complex microstructured cross-section, which adds an additional means of controlling the fiber's waveguiding characteristics. In the final section of this paper we will present some of these novel fibers and discuss their waveguiding properties.

\section{Theory and Nonlinear Characterization}

The propagation of optical pulses in our silicon optical fibers can be described using a generalized form of the nonlinear Schrödinger equation NLSE, that has been modified to account for the effects of TPA, and the TPA induced free carriers [3]:

$$
\frac{\partial A(z, t)}{\partial z}=-\frac{i \beta_{2}}{2} \frac{\partial^{2} A(z, t)}{\partial t^{2}}+i \gamma|A(z, t)|^{2} A(z, t)-\frac{1}{2}\left(\alpha_{l}+\alpha_{f}\right) A(z, t)
$$

Here $A(z, t), \beta_{2}, \gamma, \alpha_{l}$, and $\alpha_{f}$ represent the slowly varying pulse envelope, group velocity dispersion (GVD), nonlinear parameter, linear loss, and the free carrier contribution, respectively. A complex nonlinear parameter is included to account for both the Kerr and TPA contributions: $\gamma=k_{0} n_{2} / A_{\mathrm{eff}}+i \beta_{\mathrm{TPA}} / 2 A_{\mathrm{eff}}$. Similarly, the free carrier contribution is also complex: $\alpha_{f}=\alpha(1+i \mu) N_{c}$, where $\alpha$ is the free carrier absorption (FCA) coefficient and $\mu$ governs the freecarrier dispersion (FCD). The free carrier density $N_{c}$ is related to the TPA parameter and can be determined via the rate equation [3]:

$$
\frac{\partial N_{c}(z, t)}{\partial t}=\frac{\beta_{\mathrm{TPA}}}{2 h v_{0}} \frac{|A(z, t)|^{4}}{A_{\mathrm{eff}}^{2}}-\frac{N_{c}(z, t)}{\tau_{c}}
$$


where $\tau_{c}$ is the carrier life time. Fig. 1 shows the results following nonlinear characterization of a $5.7 \mu \mathrm{m}$ diameter core fiber with a linear loss determined, via cut-back measurements, of $\sim 3 \mathrm{~dB} / \mathrm{cm}$ at $1542 \mathrm{~nm}$. The TPA and FCA parameters are determined via the nonlinear absorption measurements in Fig. 1(a), where we obtain $\beta_{\text {TPA }} \sim 0.8 \mathrm{~cm} / \mathrm{GW}$ and $\sigma \sim 1 \times 10^{16} \mathrm{~cm}^{2}$ from the fitted curve. The spectral evolution displayed in Fig. 1(b) clearly demonstrates nonlinear broadening due to self-phase modulation (SPM) and fitting these spectra give an estimated Kerr coefficient of $n_{2} \sim 1.5 \times 10^{13} \mathrm{~cm}^{2} / \mathrm{W}$, as illustrated in Fig. 1(c).
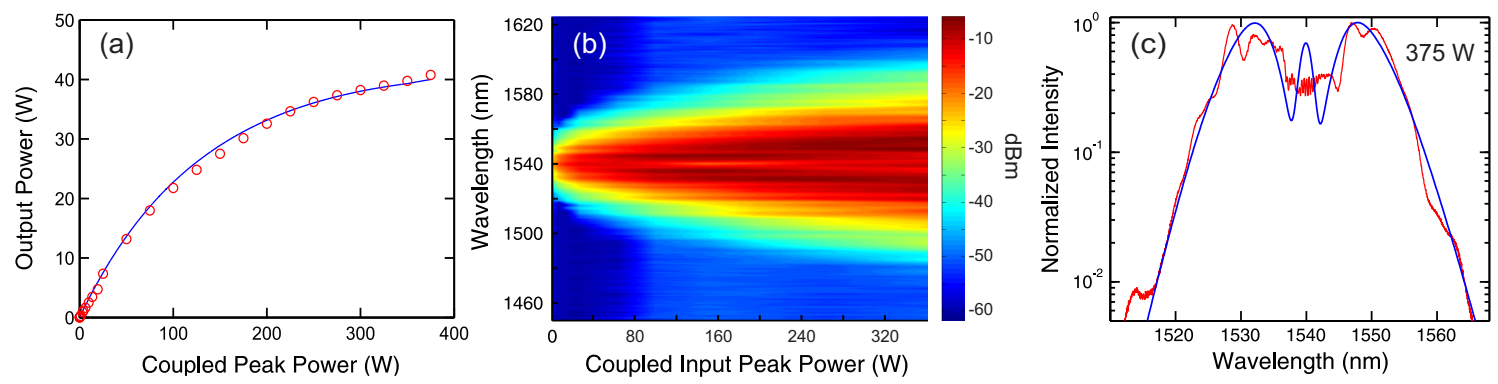

Fig. 1. Nonlinear characterization of a-Si:H fiber with a 5.7 $\mu \mathrm{m}$ diameter core. (a) Nonlinear absorption and (b) SPM as functions of input power, (c) output spectrum with NLSE fit.

More recently we have extended this type of characterization for our a-Si:H core fibers from telecoms wavelengths, across the TPA edge, and up to the edge of the mid-IR regime. The corresponding values obtained for the TPA parameter are plotted in Fig. 2(a) as a function of wavelength. These results show that $\beta_{\text {TPA }}$ initially drops sharply from $0.70 \mathrm{~cm} / \mathrm{GW}$ down to $0.28 \mathrm{~cm} / \mathrm{GW}$ as the wavelength increases through the telecoms window (1550 to 1650 $\mathrm{nm}$ ), then eventually begins to plateau to a negligible value of $0.05 \mathrm{~cm} / \mathrm{GW}$ as the wavelength approaches the mid-IR regime (1950 to $2150 \mathrm{~nm}$ ). The corresponding values of $n_{2}$ are plotted in Fig. 2(b), which shows that as the input pulse wavelength is shifted across the TPA edge the $n_{2}$ value first increases slightly up to a value of $1.75 \times 10^{-13} \mathrm{~cm}^{2} / \mathrm{W}$ at $1.75 \mu \mathrm{m}$, then drops to a value of $1.2 \times 10^{-13} \mathrm{~cm}^{2} / \mathrm{W}$ at $2150 \mathrm{~nm}$. The nonlinear figure of merit $\left(\mathrm{FOM}_{\mathrm{NL}}=n_{2} / \beta_{\mathrm{TPA}} \lambda[2]\right)$ can be used to determine a materials suitability for a particular nonlinear application. We have used our values of the nonlinear parameters $\beta_{\text {TPA }}$ and $n_{2}$ to investigate the dispersion of the FOM $\mathrm{NL}_{\mathrm{NL}}$, plotted in Fig. 2(c). This figure clearly shows that despite the decrease in $n_{2}$ at the longer wavelengths, the dramatic reduction in $\beta_{\text {TPA }}$ results in a monotonic increase in the $\mathrm{FOM}_{\mathrm{NL}}$. Thus, although the value of the $\mathrm{FOM}_{\mathrm{NL}}$ at $1550 \mathrm{~nm}$ for this a-Si:H core material is comparable to what we have reported before $\sim 1.6$, it increases rapidly to $\sim 10$ for the peak value of $n_{2}$ at $1750 \mathrm{~nm}$, then even further up to $\sim 28$ at the longest wavelengths. The results suggest that a-Si:H waveguides are a viable platform for nonlinear applications extending beyond telecoms, and into the short wavelength end of the mid-IR regime where applications include free-space communications, gas detection and medical diagnostics.
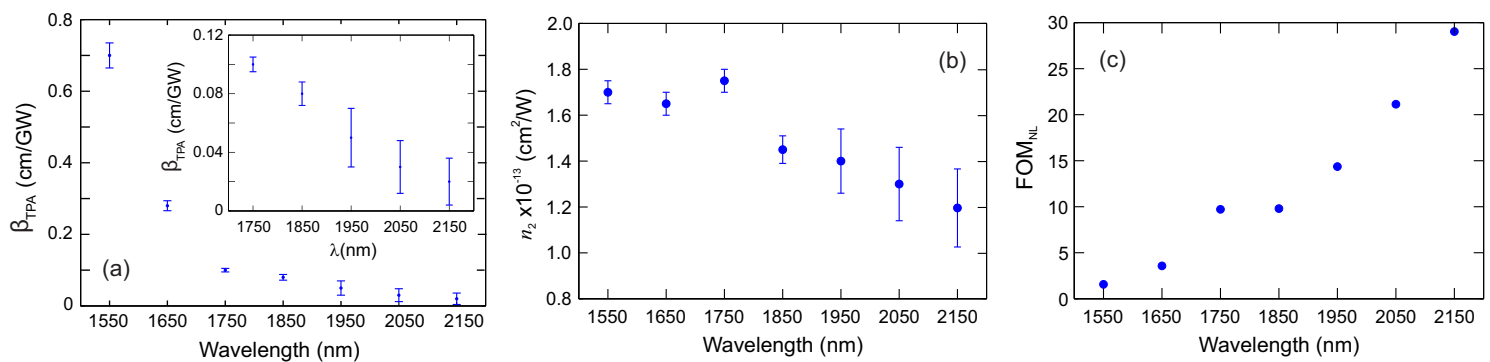

Fig. 2. (a) TPA parameter as a function of wavelength.(a). Inset: close up of the low value $\beta_{\text {TPA }}$ region. (b) Wavelength dependence of the Kerr nonlinear coefficient $n_{2}$. (c) Wavelength dispersion of the $\mathrm{FOM}_{\mathrm{NL}}$. Error bars represent the uncertainty in the input powers. 


\section{Microstructured Geometries}

By incorporating high refractive index semiconductor materials into the low index voids of microstrucured optical fiber (MOF) templates we can transform the waveguiding mechanism from modified total internal reflection (MTIR) to photonics bandgap (PBG) and vice versa. The flexibility of our HPCVD approach makes it possible to fabricate silicon versions of these optical fibers even for templates with nanoscale silica microstructures. In an attempt to suppress the number of guided modes in the micron sized diameter semiconductor cores, we have fabricated a fiber with a reduced core/cladding index contrast by incorporating additional semiconductor material into the pores of a microstructured cladding. This large mode area (LMA) fiber was formed by completely filling a hollow core silica PBG fiber with silicon. The resulting fiber, shown in Fig. 3(a), has a high index core and the lattice of silicon rods and nanoscale silica webbing acts as a complex cladding that disrupts the propagation of the higher order modes. This fiber had a core of $8 \mu \mathrm{m}$ and supported just two modes, see Fig. 3(b).
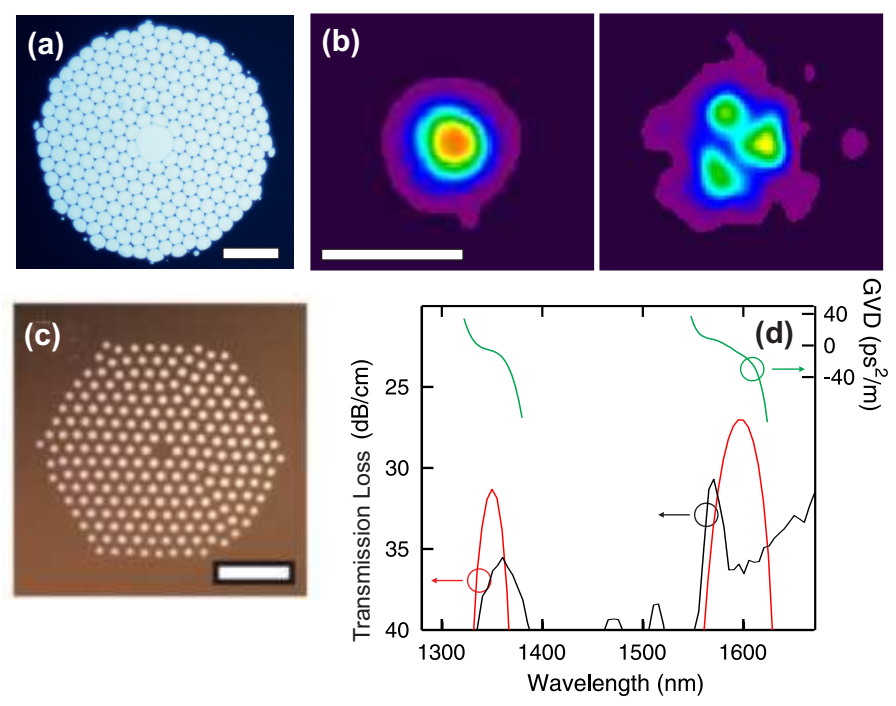

Fig. 3. (a) LMA silicon fiber. (b) Modes supported by LMA silicon fiber. (c) Micrograph of a Silicon ARROW fiber. (d) Measured (black) and numerically simulated (red) transmission spectra of the SiARROW fibre in (c), together with the simulated GVD curve (green). Scale bars $10 \mu \mathrm{m}$.

By doing the reverse, filling a MTIR fiber with a-Si:H and converting it to a PBG fiber with a solid silica core it is possible to decouple the functionality of the semiconductor from its material losses and high input/output reflectivity. To this end, we have fabricated the first antiresonant reflecting optical waveguiding (ARROW) silicon fiber, where the light is guided in the low loss silica core surrounded by high index silicon inclusions. This fiber was fabricated by filling the holes of a solid core silica MOF with a-Si:H, as illustrated in Fig. 3(c). The MOF template had a cladding hole diameter of $d=1.17 \mu \mathrm{m}$ and a triangular lattice with a pitch of $\Lambda=2.15 \mu \mathrm{m}$. The guiding mechanism functions such that a mode cannot be confined in the core if it is on resonance with one of the modes of a cladding inclusion, while conversely, if the light is off resonance with the inclusions it is reflected back into the core where it is guided. This resonance based guidance means that the ARROW fiber has a transmission band guidance structure and that the position and width of these bands are highly dependent on the size and refractive index of the inclusions. This structure along with the numerical simulations of the fiber's guidance properties are shown in Fig. 3(d).

\section{References}

1. L. Lagonigro, N. Healy, J. R. Sparks, N. F. Baril, P. J. A. Sazio, J. V. Badding, and A. C. Peacock, "Low loss silicon fibers for photonics applications," Appl. Phys. Lett. 96, 041105 (2010).

2. P. Mehta, N. Healy, N. F. Baril, P. J. A. Sazio, J. V. Badding, and A. C. Peacock, "Nonlinear transmission properties of hydrogenated amorphous silicon core optical fibers," Opt. Express 18, 16826-16831 (2010).

3. L. Yin and G. P. Agrawal, "Impact of two-photon absorption on self-phase modulation in silicon waveguides," Opt. Lett. 32, 2031-2033 (2007). 